\title{
Le cannabis : quelle place dans la soumission chimique?
}

\section{Cannabis : which occurence in chemical submission?}

\section{Patrick MURA*(1), Pascale VISINONI ${ }^{(2)}$, Jean-Claude ALVAREZ ${ }^{(3)}$, Jean-Pierre GOULLÉ(4), Pascal KINTZ ${ }^{(5)}$}

(1) Laboratoire de Biochimie et Toxicologie, Centre Hospitalier Universitaire - BP 577 - 86021 POITIERS

(2) Laboratoire de Police Scientifique, 23, Boulevard de l'Embouchure - 31021 TOULOUSE

(3) Centre Hospitalier Général, 104, Boulevard Poincaré - 92380 GARCHES

(4) Centre Hospitalier Général - BP 24 - 76083 LE HAVRE

(5) Institut de Médecine Légale, 11, rue Humann - 67085 STRASBOURG

* Auteur à qui adresser la correspondance : Patrick MURA, Laboratoire de Biochimie et Toxicologie, Centre Hospitalier Universitaire, BP 577 - 86021 POITIERS

(Reçu le 14 octobre 2002 ; accepté le 28 octobre 2002)

\section{RÉSUME}

Le cannabis est de très loin la drogue illicite la plus consommée en France. Les effets chez l'homme sont dus au delta-9tétrahydrocannabinol (THC) et au 11-hydroxy-delta-9-tétrahydrocannabinol (11-OH-THC). Les effets aigus consistent en une euphorie, une désinhibition, un état de somnolence pouvant aller jusqu'à un sommeil profond, des troubles visuels et des troubles de la mémoire à court terme. Avec de tels effets, il n'est pas surprenant que le cannabis puisse être présent dans l'organisme des auteurs et/ou victimes de viols, vols, ou autres crimes faisant appel à la soumission chimique. Cette potentialité n'est cependant pas confirmée par l'expérience des auteurs puisque, parmi plusieurs centaines d'expertises judiciaires réalisées au cours des trois dernières années dans des cas de suspicion de viol, des cannabinoïdes étaient présents dans le sang et/ou les urines de seulement 21 victimes supposées. Pour 9 d'entre-elles, l'analyse des cheveux n'ayant pas été réalisée, la soumission chimique n'a pas pu être confirmée. L'analyse des cheveux a permis de

\section{SUMMARY}

Cannabis is largely the major drug of abuse consumed in France. Effects in human are due to delta-9 tetrahydrocannabinol (THC) and 11-hydroxy delta-9-tetrahydrocannabinol (11-OH-THC). The acute effects are euphoria, desinhibition, drowsiness up to heavy sleep, visual disorders and short-memory troubles. With such effects, it is not surprising that cannabis may be present in the organism of authors andlor victims of rapes, robberies or others crimes using chemical submission. This potentiality is not confirmed by our experience since among several hundreds of toxicological investigations realized during the last three years in sexual assaults cases, cannabinoids were only present in blood and/or urines of 21 subjects. In 9 of these positive cases, since hair analysis was not performed, it was not possible to conclude. In 11 cases, hair analysis revealed a chronic cannabis use. In only one case, THC and 11-OH THC were present in blood whereas hair was free of cannabinoids, confirming the hypothesis of the use of cannabis for 
mettre en évidence une consommation chronique de cannabis dans 11 cas. Dans un seul cas, la présence de THC et de 11-OH-THC dans le sang était associée à l'absence de cannabinoides dans les cheveux, permettant ainsi de conforter l'hypothèse d'une utilisation du cannabis à des fins criminelles. Les auteurs en concluent que toute recherche positive de cannabinoïdes dans le sang et/ou les urines doit être suivie d'une analyse des cheveux, qui doivent donc être systématiquement prélevés.

\section{Introduction}

Si certains effets du cannabis (manifestations cardiovasculaires, effets sur le système immunitaire, pouvoir mutagène) sont encore discutés, les perturbations neuropsychiques induites par une consommation de cannabis sont désormais admises par tous les auteurs (1). C'est en raison de ces effets qu'un usage récent de cannabis peut avoir des conséquences néfastes pour autrui dans le cas des conducteurs d'automobile (2) ou des personnes occupant des postes de sécurité en entreprise (3). Dans ce chapitre de la toxicité pour autrui, l'implication du cannabis dans la soumission chimique mérite d'être étudiée.

Les principaux effets recherchés lorsqu'une substance est utilisée dans un but de soumission chimique (administration de substances psychoactives à une personne à des fins délictueuses ou criminelles), consistent en une désinhibition, une altération des fonctions cognitives et psychomotrices, des troubles de la mémoire. C'est pourquoi l'acide gamma-hydroxybutyrique (GHB), l'alcool éthylique, certaines benzodiazépines et des hypnotiques comme le zolpidem semblent être parmi les produits les plus utilisés $(4,5)$. Le cannabis est-il responsable de tout ou partie de ces effets?

Pour caractériser une éventuelle soumission chimique, les experts judiciaires sont amenés à rechercher dans les milieux biologiques des victimes et/ou des auteurs la présence de toute substance psychoactive. Le cannabis est-il fréquemment retrouvé dans ces expertises ?

En France, la consommation de cannabis est en augmentation constante. Les résultats de l'enquête réalisée en 2001 par l'Observatoire Français des Drogues et des Toxicomanies auprès des jeunes de 18 ans à l'occasion de la journée d'appel de préparation à la défense révèlent que plus d'un jeune sur deux a déjà consommé du cannabis et que $20 \%$ des garçons en ont consommé plus de 10 fois au cours du dernier mois. Ce produit est donc aujourd'hui très facilement accessible sur le marché clandestin. Une étude récente reposant sur des analyses sanguines a confirmé cette importante prévalence de consommation puisque chez $9 \%$ des moins de 27 ans (sujets hospitalisés pour une cause médicale non programmée), du principe actif du cannabis était the purpose of a criminal act. The authors conclude that each positive cannabis result in blood or urine has to be followed by hair analysis, a speciem which must be systematically sampled.

retrouvé dans le sang. Dans les cas de soumission chimique pour lesquels du cannabis était présent dans le sang, les victimes étaient-elles consommatrices de ce produit?

\section{Les mécanismes impliqués au niveau cérébral.}

Les principes actifs du cannabis sont le delta-9-tétrahydrocannabinol (THC) et le 11-hydoxy-delta-9-tétrahydrocannabinol (11-OH-THC). Si les mécanismes d'action concernant le 11-OH-THC sont mal connus, ceux du THC sont en revanche aujourd'hui bien établis (6). Les cibles cellulaires du THC sont les récepteurs CB1. $\mathrm{Au}$ niveau du cerveau, on les retrouve en très grand nombre dans les structures suivantes:

- le ganglion basal et le cervelet. Ces deux zones sont très largement impliquées dans la motricité et le contrôle postural.

- le cortex frontal, impliqué dans la vision, le goût, les capacités de concentration mentale. Des dysfonctionnements à ce niveau sont responsables de distorsions caractéristiques de la notion du temps et de l'espace, de difficultés à se concentrer et de l'apparition d'un état rêveur.

- l'hippocampe, impliqué dans les phénomènes de mémorisation et de codage des informations sensorielles.

Les mécanismes d'action du THC au niveau des neurones sont illustrés par la Figure 1 (7). En l'absence de THC, le récepteur est couplé à une protéine $\mathrm{G}$ inhibitrice. L'adénylate cyclase, une enzyme membranaire, joue pleinement son rôle en favorisant la synthèse d'AMP cyclique. Ce dernier intervient en activant une protéine kinase qui permet de maintenir le canal potassique en position fermée. Les ions potassium restent ainsi à l'intérieur du neurone.

En présence de THC, la protéine $\mathrm{G}$ inhibitrice est «larguée» et n'est donc plus accolée au récepteur. Elle va alors chercher un autre site de fixation et trouve l'adénylate cyclase. En s'y accolant, elle inhibe sa fonction. La synthèse d'AMP cyclique est alors arrêtée, et il n'y 
a plus d'activation de la protéine kinase. Par voie de conséquence, le canal potassique reste en position ouverte, et on assiste à une fuite du potassium vers l'extérieur du neurone.

D'autres systèmes de transduction interviennent, conduisant à un accroissement des concentrations intracellulaires de calcium.

Toutes ces modifications métaboliques sont responsables de dysfonctionnements neuronaux dont une inhibition du relargage de certains neurotransmetteurs comme le glutamate, en diminuant les capacités d'exocytose.

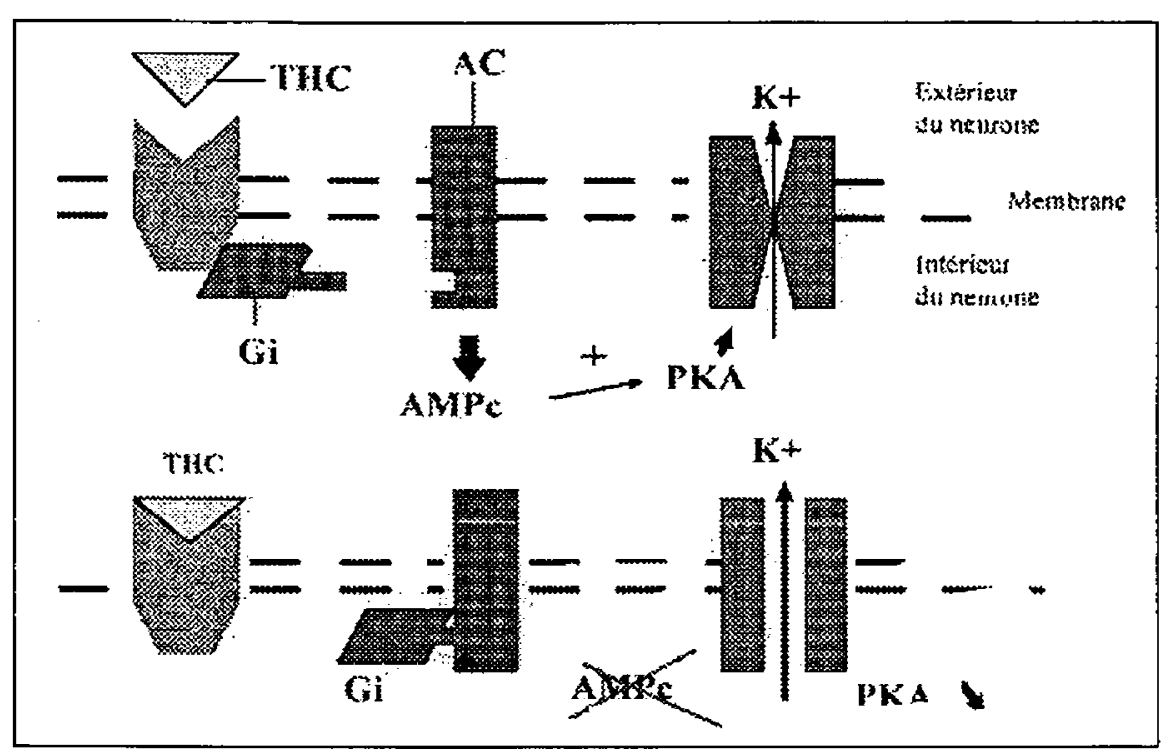

Figure 1 : Schéma du mécanisme d'action du THC conduisant au maintien du canal potassique en position ouverte.

$T H C$ : delta-9 tétrahydrocannabinol, $R$ : récepteur au cannabis $C B l, G i$ : protéine $G$ inhitrice, $A C$ : Adénylate cyclase, $A M P c: A M P$ cyclique, $P K A$ : Protéine kinase active, $K+:$ Ion potasium.

\section{Les effets aigus sur le consommateur.}

Nous ne décrirons ici que les effets pouvant être impliqués dans le cadre de la soumission chimique.

Lors d'un usage occasionnel, l'intoxication aiguë au cannabis se caractérise par :

$>$ des troubles de la pensée avec désorientation temporelle,

- des troubles mnésiques concernant la mémoire à court terme, pouvant persister pendant plusieurs jours,

$>$ des perturbations sensorielles avec une exacerbation sensitive associée à des troubles de la vue,

$>$ des troubles thymiques et dissociatifs avec euphorie, anxiété, agressivité, dépersonnalisation, hallucinations et délire dans le cas de doses élevées,

$>$ des performances intellectuelles et motrices altérées, avec une diminution de la vigilance voire un endormissement profond.
$>$ Une désinhibition importante.

Ces effets sont cependant variables selon la dose, la tolérance du sujet et l'association éventuelle à l'alcool ou à des médicaments psychoactifs.

Diminution de la vigilance, désinhibition, troubles visuels, amnésie antérograde, sont autant d'effets pouvant «intéresser» les auteurs de tels crimes.

\section{Les modes d'administration}

Le cannabis est consommé par inhalation ou par ingestion.

En ce qui concerne l'inhalation, elle est généralement réalisée en fumant un mélange «d'herbe» ou de «résine» avec du tabac, soit dans du papier à cigarette («pétard» ou «joint») soit à l'état pur (pipes à kif). Certains consommateurs utilisent des narguilés, pipes orientales à long tuyau communiquant avec un flacon d'eau aromatisée que la fumée traverse avant d'être inhalée. Avec ces modes de consommation, il va de soi que cela ne peut pas se faire à l'insu de la victime. Il peut cependant s'agir d'une incitation à la consommation, qui peut aussi caractériser la soumission chimique.

Le cannabis sous sa forme «résine» ou «haschish» peut être aussi consommé en l'incorporant à des aliments comme des pâtisseries au chocolat. De cette manière, le cannabis peut donc être administré à l'insu de la victime.

\section{Analyse de 27 cas}

Afin de savoir si le cannabis était souvent utilisé à des fins criminelles dans le cadre de la soumission chimique, les auteurs ont analysé les résultats de 27 cas «positifs».

\section{Matériel et méthodes}

Dans tous les cas, il s'agissait de réquisitions judiciaires effectuées dans des affaires de suspicion de viol. Les échantillons biologiques étaient constitués de sang, associé ou non à un échantillon d'urines et/ou de cheveux.

Les principes actifs (THC et 11-OH THC) et le métabolite inactif (THC-COOH) du cannabis étaient identifiés et dosés dans le sang et les urines par la méthode recommandée par la Société Française de Toxicologie Analytique (8). Le seuil analytique utilisé était de 0,2 $\mathrm{ng} / \mathrm{ml}$. Dans les cheveux, l'analyse chromatographique était précédée par une étape de décontamination externe $(9-10)$. 


\section{Prévalence}

Il est à noter que, sur la base des analyses sanguines, la prévalence observée n'est pas très élevée et semble être très variable selon les régions. Ainsi par exemple, parmi les 42 expertises réalisées chez des auteurs ou victimes de viols de janvier 2000 à février 2002 à l'Institut de Médecine Légale de Strasbourg, du THC était présent dans le sang dans 9 cas, soit $21,4 \%$. A l'opposé, parmi les 24 expertises judiciaires pour suspicion de viol réalisées pendant la même période au Centre Hospitalier Universitaire de Poitiers, un seul cas (4\%) était observé.

\section{Absence de THC et de 11- $\mathrm{OH}$ THC (sang), présence de THC-COOH (sang et/ou urines)}

Ce résultat a concerné 10 sujets ( $37 \%$ ) dont 7 victimes et 3 auteurs. L'alcool était associée dans 2 cas.

La seule conclusion pouvant être apportée est : les sujets n'étaient pas sous influence de cannabis au moment du prélèvement.

Cependant, cela pose le problème du délai écoulé entre les faits et le prélèvement, qui est souvent de plusieurs heures voire plusieurs jours. Bien évidemment, lorsque plusieurs jours se sont écoulés, l'analyse sanguine ne peut plus confirmer une éventuelle consommation récente de substances psycho-actives. En ce qui concerne le cannabis, quelques heures suffisent pour que les principes actifs aient disparu du compartiment sanguin (11).

\section{Présence de THC et de 11- $\mathrm{OH}$ THC dans le sang}

La présence de principe(s) actif(s) dans le sang a été observée dans 17 cas $(63 \%)$ correspondant à 14 victimes et 3 auteurs. Les concentrations variaient de 0,5 à $13 \mathrm{ng} / \mathrm{ml}$, avec une moyenne de $4,1 \mathrm{ng} / \mathrm{ml}$.

Le cannabis était seul présent dans 4 cas (23\%), associé à l'alcool dans 11 cas $(65 \%)$, à des benzodiazépines dans 2 cas. L'association avec un autre stupéfiant était observée dans 2 cas ( 1 avec la morphine, l'autre avec de la MDMA).

La présence de principe(s) actif(s) du cannabis dans les échantillons sanguins (quelque soit les concentrations) nous permet d'estimer que ces sujets étaient sous influence de cannabis au moment du prélèvement (12). Mais, comment être sûr que le cannabis a été utilisé à des fins criminelles ? En d'autres termes, les sujets concernés n'étaient-ils pas des consommateurs réguliers?

\section{Les résultats des analyses de cheveux chez les vic- times}

L'analyse des cheveux permet de mettre en évidence une consommation chronique (10). La vitesse moyenne de pousse étant de $1 \mathrm{~cm}$ par mois, une analyse par segments de $1 \mathrm{~cm}$ permet de préciser l'évolution de la consommation au cours des derniers mois.

Parmi les 21 victimes chez lesquelles des cannabinoïdes étaient présents dans le sang, l'analyse des cheveux n'a pas été réalisée dans 9 cas. Pour ces 9 «victimes», les analyses toxicologiques ne permettent pas d'argumenter sur l'utilisation du cannabis à des fins criminelles.

La recherche et le dosage des cannabinoïdes dans les cheveux, réalisée pour les 12 autres victimes supposées, a révélé une consommation régulière de cannabis dans 11 cas. Là encore, il est permis de douter quant à l'administration ou l'incitation à la consommation de ce produit à des fins criminelles.

L'absence de cannabinoïdes dans les cheveux associée à la présence de THC et de 11-OH dans le sang a été observée dans un seul cas parmi les 21 victimes présumées.

Parmi les centaines d'expertises toxicologiques réalisées par l'ensemble des auteurs au cours de ces dernières années, il s'agit donc du seul cas suffisamment documenté analytiquement pour lequel, dans la conclusion de l'expert judiciaire, le cannabis peut être considéré comme l'instrument chimique de l'acte criminel.

\section{Conclusion}

En raison de ses effets neuropsychiques, il n'est pas surprenant que le cannabis puisse être présent dans l'organisme des auteurs et/ou victimes de viols, vols, ou autres crimes pouvant faire appel à la soumission chimique. Bien que cette étude montre que l'utilisation du cannabis à des fins de soumission chimique semble aujourd'hui peu fréquente, il n'en demeure pas moins que, compte tenu de l'augmentation de son accessibilité en France, il doit systématiquement être recherché lors des investigations toxicologiques. D'autre part, notre étude confirme que toute recherche de cannabis positive dans le sang et/ou les urines doit être suivie d'une analyse des cheveux, qui devraient donc être systématiquement prélevés. 


\section{Références}

1. Expertise collective INSERM. Cannabis. Quels effets sur le comportement et la santé ? In : INSERM, ed. Paris : Jouve, 2001.

2. Mura P. Accidentologie et drogues illicites. Bull Acad Natle Méd $2002 ; 186: 345-57$.

3. Mura P. Cannabis sativa var. indica : une menace croissante pour les entreprises. Annales de Toxicologie Analytique $2002 ; 14: 64-67$.

4. Deveaux M. Les pièges du GHB. Anales de Toxicologie Analytique $2002 ; 13: 294-295$.

5. Kintz P. Soumission chimique : aspects pratiques en vue d'une expertise toxicologique. Annales de Toxicologie Analytique $2002 ; 13: 120-121$.

6. Julien RM. Ed. A Primer of Drug Action. New York : WH Freeman and Company, 1997.

7. Mura P., Piriou A. Le cannabis. In : Mura P., ed. Alcool, médicaments, stupéfiants et conduite automobile. Paris : Elsevier, 1999 ; 143-169.
8. Kintz P., Cirimele V., Pepin G., Marquet P., Deveaux M., Mura P. Identification et dosage des cannabinoïdes dans le sang total. Toxicorama $1996 ; 8: 29-33$.

9. Kintz P., Mangin P. What constitutes a positive result in hair analysis : proposal for the establishment of cut-off values. Forensic Sci. Int. $1995 ; 70: 3-11$.

10.Cirimele V. Cannabis and amphetamine : determination in human hair. In : Kintz P., ed. Drug testing in hair. Boca Raton : CRC Press, 1996 ; 181-9.

11. Huestis M, Henningfield J, Cone E. Blood cannabinoids.II. Models for the prediction of time of marijuana exposure from plasma concentrations of THC and THCCOOH. J Anal Toxicol $1992 ; 16: 283-286$.

12. Mura P., Papet Y., Baritault N. Cannabis et conduite automobile : une valeur sanguine limite est-elle nécessaire ? Annales de Toxicologie Analytique $2002 ; 14: 149-150$. 